\title{
Correlation of fecal calprotectin and patient-reported outcome measures in patients with ulcerative colitis
}

\author{
Nagesh Kamat, Sudheer K Vuyyuru, Saurabh Kedia, Pabitra Sahu, Bhaskar Kante, Peeyush Kumar, \\ Mukesh Kumar Ranjan, Mukesh Kumar Singh, Sambuddha Kumar, Vikas Sachdev, Govind Makharia, Vineet Ahuja \\ Department of Gastroenterology and Human Nutrition, All India Institute of Medical Sciences, New Delhi, India
}

Ulcerative colitis (UC) is a chronic immune-mediated inflammatory disorder of unknown etiology causing significant impairment in quality of life and negatively affects an individual's psychological well-being. There are many indices developed to assess disease activity like the Mayo Clinic score, Baron score, Ulcerative Colitis Endoscopic Index of severity (UCEIS), Simple Clinical Colitis Activity Index (SCCAI). However, these scores are developed by clinicians not considering patient opinions. Patient-reported outcomes (PROs) are validated questionnaires used to measure various aspects of health, including physical, emotional, or social domains. The Food and Drug Administration (FDA) defined PRO as "any report of the status of a patient's health condition that comes directly from the patient, without interpretation of the patient's response by a clinician or anyone else."2 PROs developed till now are complex, time taking and difficult to use in regular clinical practice. Recently FDA mandated the inclusion of clinical outcome assessment (COA) as either co-primary or secondary endpoint in clinical trials and issued guidance on the development and validation of $\mathrm{COA}{ }^{3} \mathrm{COA}$ includes 4 components which include PROs, clinician-reported outcomes, observer reported outcomes, and performance outcomes. The development of a valid PRO is a rigorous, tedious process involving patient interviews, expert interviews, item generation, assessing for con-

Received April 25, 2021. Revised June 21, 2021. Accepted June 23, 2021. Correspondence to Vineet Ahuja, Department of Gastroenterology and Human Nutrition, All India Institute of Medical Sciences, Room No. 3093, Third Floor, Teaching Block, New Delhi 110029, India. Tel: +91-11-26593300, Fax:+91-11-2658663, E-mail: vineet.aiims@gmail.com tent validity, construct validity and subsequently further validation. The inflammatory bowel disease control (IBD control) questionnaire is one such questionnaire developed and validated according to the recommended guidelines. ${ }^{4}$ Even though recently there has been an increasing demand for the use of PROs, their role in predicting mucosal activity is not clear. Fecal calprotectin (FCP) is a sensitive diagnostic marker for disease activity in UC, ${ }^{5}$ and numerous studies have shown a significant correlation of FCP with endoscopic and histological healing. Recently updated Selecting Therapeutic Targets in Inflammatory Bowel Disease (STRIDE) consensus also recommends inflammatory markers like C-reactive protein (CRP) and FCP as intermediate targets. There are no studies to show the correlation of PROs with inflammatory markers like FCP. Hence, we designed this cross-sectional study to demonstrate the correlation between PROs and FCP.

We included consecutive patients with UC of any disease extent and severity under follow-up at All India Institute of Medical Sciences, New Delhi, India. Patients diagnosed with UC for at least 6 months, $>18$ years of age, were included. This study was conducted in accordance with Good Clinical Practice and in a manner to conform with the Helsinki Declaration of 1975, as revised in 2000 and 2008 concerning human rights. The institutional ethics committee approved the study protocol (AIIMS IRB No. IESC/T-277), and all patients gave their written informed consent before inclusion. A detailed evaluation was done for demographics, disease duration, extent and activity. Disease extent was classified on the basis of Montreal classification. The primary objective of this study was to assess 
the correlation between FCP and PRO (IBD control-8 and IBD control-VAS), and the secondary objective was to assess the diagnostic performance of FCP and PRO in identifying clinical remission.

Clinical remission was assessed by the clinician-based SCCAI score. SCCAI has 6 components: bowel frequency (day and night), blood in stools, urgency, general well-being, and extraintestinal manifestations. The score ranges from 0 to 20 . Clinical remission was defined as an SCCAI score of less than or equal to $2{ }^{6}$

The IBD-control questionnaire was selected as it was the first PRO developed considering the patient's perspective. It contains 2 components: visual analog scale (VAS) ranging from 0 to 100 and IBD control-8 subscore. Original IBD control questionnaire is comprised of 13 items, each with 3 possible answers ("no," “yes," and "not sure" or "better," "worse" and "no change"). IBD control-8 questionnaire contains 8 items from the original 13 items, and the score ranges from 0 to 16 (Supplementary Table 1).

FCP was analyzed by collecting 5-10 $\mathrm{g}$ of the first stool sample of the day and stored at $-80^{\circ} \mathrm{C}$. FCP levels were measured by fluorescence-immunoassay (EliA) 2-site sandwich technique (Phadia-250; Thermo Fisher Scientific, Waltham, MA, USA) as per the protocol mentioned by the manufacturer. The laboratory technician was blinded to patients' details. FCP concentration was calculated from the standards and expressed as $\mu \mathrm{g} / \mathrm{g}$.

Statistical analysis was done by SPSS version 21.0 for Windows (IBM Corp., Armonk, NY, USA). Descriptive statistics were used wherever appropriate. The area under the curve (AUC) with the 95\% confidence interval was studied. Using the AUC, the sensitivity and specificity of the cutoff point were calculated. Mann-Whitney, chi-square, and $t$-test were used for quantitative and qualitative variables. Correlations between clinical activity indices and FCP were made by the non-parametric 2-tailed Spearman's rank correlation coefficient when the variables were not normally distributed. $P<0.05$ was considered statistically significant.

Out of the 96 patients screened, 57 were included in the final analysis after excluding 39 patients (Supplementary Fig. 1). The mean age at enrollment of the cohort was $37.5 \pm 12.0$ years, and 58\% were male. The median disease duration was 60 months (range, $36-108$ months). Nine patients (15.8\%) had proctitis (E1), 26 (45.6\%) had left-sided colitis (E2), and $22(38.6 \%)$ had extensive colitis (E3). Fourth-three patients (75.4\%) belonged to urban areas. Ninety-one percent were on 5-amino salicylate (ASA), 38.6\% were on topical ASA, 15.8\% on steroids, $24.6 \%$ on topical steroids, $31.6 \%$ on azathioprine, and $1.8 \%$ on biologics. The median FCP of the entire cohort was 148 (interquartile range [IQR], 23-1,283). FCP was $<150$ in $50.8 \%(\mathrm{n}=29)$ patients (Table 1$)$.

Of the entire cohort, $56.1 \%(n=32)$ were in clinical remis-

Table 1. Baseline Characteristics of Patients with Ulcerative Colitis

\begin{tabular}{|c|c|c|c|c|}
\hline Characteristics & All & $\begin{array}{c}\text { Remission } \\
(n=32)\end{array}$ & $\begin{array}{l}\text { Active } \\
\text { disease } \\
(n=25)\end{array}$ & $P$-value \\
\hline Age at enrollment (yr) & $37.5 \pm 12.0$ & $37.3 \pm 11.0$ & $37.8 \pm 13.5$ & 0.889 \\
\hline Sex & & & & 0.426 \\
\hline Male & 33 (57.9) & $20(62.5)$ & $13(52.0)$ & \\
\hline Female & $24(42.1)$ & $12(37.5)$ & $12(48.0)$ & \\
\hline Region & & & & 0.931 \\
\hline Rural & $14(24.6)$ & $8(25)$ & $6(24.0)$ & \\
\hline Urban & $43(75.4)$ & $24(75)$ & $19(76.0)$ & \\
\hline Socioeconomic status ${ }^{\mathrm{a}}$ & & & & 0.398 \\
\hline Low & $10(17.5)$ & $7(21.9)$ & $3(12.0)$ & \\
\hline Middle & $10(17.5)$ & 4 (12.5) & $6(24.0)$ & \\
\hline Upper & $37(65.0)$ & $21(65.6)$ & $16(64.0)$ & \\
\hline Alcohol intake & & & & 0.741 \\
\hline Non alcoholic & $43(75.4)$ & $25(78.1)$ & $18(72.0)$ & \\
\hline Former alcoholic & $9(15.8)$ & 5 (15.6) & $4(16.0)$ & \\
\hline Current alcoholic & $5(8.8)$ & $2(6.3)$ & $3(12.0)$ & \\
\hline Smoking status ${ }^{c}$ & & & & 0.492 \\
\hline Non smoking & $53(93)$ & 30 (93.8) & 23 (92.0) & \\
\hline Former smoker & $1(1.8)$ & $1(3.1)$ & 0 & \\
\hline Current smoker & $3(5.3)$ & $1(3.1)$ & $2(8.0)$ & \\
\hline Diet & & & & 0.898 \\
\hline Vegetarian & $20(35.1)$ & $11(34.4)$ & $9(36.0)$ & \\
\hline Non vegetarian & $37(64.9)$ & 21 (65.6) & $16(64.0)$ & \\
\hline Disease extent & & & & 0.420 \\
\hline E1 & $9(15.8)$ & 6 (18.8) & $3(12.0)$ & \\
\hline E2 & $26(45.6)$ & $16(50.0)$ & $10(40.0)$ & \\
\hline E3 & $22(38.6)$ & 10 (31.3) & $12(48.0)$ & \\
\hline
\end{tabular}

Values are presented as mean \pm standard deviation or number (\%). ${ }^{a}$ Socioeconomic status is categorized based on Agarwal classification system. ${ }^{7}$ ${ }^{b}$ Current alcoholic: consuming 15 or more standard drinks per week or 5 or more on an occasion, for men, or 8 or more drinks weekly or 4 or more on an occasion, for women and people older than 65 years of age; previous alcoholic: abstained from alcohol for more than 1 year.

'Current smoker: an adult who has smoked 100 cigarettes in his or her lifetime and who currently smokes cigarettes; former smoker: an adult who has smoked at least 100 cigarettes in his or her lifetime but who had quit smoking at the time of interview. 
sion based on the SCCAI score. The number of patients in clinical remission was similar across different extents of UC (E1 vs. E2 vs. E3). The median FCP in patients with clinical remission was 37 (IQR, 16-195), whereas, in patients with active disease, it was 1,343 (IQR, 519-3,000). The mean IBD control-8 score of the entire cohort was 11.6 \pm 3.9 . Patients in clinical remission had a mean score of 13.5 compared to 9 in patients with active disease $(P<0.001)$. The median IBD controlVAS score of the entire cohort was 60 (IQR, 50-80), whereas it was 80 in patients with remission compared to 50 patients with active disease $(P<0.001)$. There was a significant negative correlation between FCP and IBD control-8 $(-0.57 ; P<0.001)$. Similarly, the correlation between FCP and IBD-VAS score $(-0.40 ; P<0.001)$ was also significant. Correlation between FCP and individual items of the IBD control-8 questionnaire was also done. Among all questions, Q1a had strongest correlation $(-0.5 ; P<0.001)$ (Supplementary Table 2).

We also assessed the correlation and diagnostic performance of FCP, IBD control-8 and IBD control-VAS in identifying clinical remission. There was a significant positive correlation between FCP and SCCAI score $(0.65 ; P<0.001)$. FCP cutoff of $150 \mu \mathrm{g} / \mathrm{gm}$ had a sensitivity of $81.3 \%$ and specificity of $88 \%$ in identifying patients in clinical remission with an AUC of 0.88 (95\% confidence interval, $0.77-0.97 ; P<0.001$ ) (Supplementary Fig. 2). The IBD control-8 and IBD control-VAS scores had significant negative correlation with SCCAI, $(-0.59 ; P<0.001)$ and $(-0.60 ; P<0.001)$, respectively. IBD control-8 had moderate diagnostic accuracy in identifying patients in clinical remission (area under curve, 0.87 [0.78-0.97]). A cutoff of 13 points had a sensitivity and specificity of $71.8 \%$ and $88 \%$ in identifying patients in clinical remission. In comparison, IBD control VAS score of $\geq 85$ had good specificity (92\%) but poor sensitivity (34.4\%) in identifying clinical remission (Table 2, Fig. 1).

UC is an idiopathic inflammatory disorder, and poorly controlled disease leads that to poor quality of life. ${ }^{8}$ Outcome mea-

Table 2. Diagnostic Accuracy of FCP, IBD Control-8, and IBD Control-VAS for Predicting Clinical Remission

\begin{tabular}{lccc}
\hline Variable & $\begin{array}{c}\text { AUC } \\
(95 \% \mathrm{Cl})\end{array}$ & $\begin{array}{c}\text { Specificity } \\
(\%)\end{array}$ & $\begin{array}{c}\text { Sensitivity } \\
(\%)\end{array}$ \\
\hline FCP & $0.88(0.77-0.97)$ & 88.0 & 81.3 \\
IBD control-8 & $0.87(0.78-0.97)$ & 88.0 & 71.8 \\
IBD control-VAS & $0.83(0.72-0.94)$ & 92.0 & 34.4 \\
\hline
\end{tabular}

FCP, fecal calprotectin; IBD, inflammatory bowel disease; VAS, visual analog scale; $\mathrm{AUC}$, area under the curve; $\mathrm{Cl}$, confidence interval. sures like endoscopic healing, biomarkers and histopathological improvement determine the future course of the disease, and the clinicians tend to give more importance to them, disregarding the patient's perception of disease control. A survey by Schreiber et al. ${ }^{9}$ has demonstrated a discrepancy between clinician and patient's perception of disease severity. Hence, FDA has issued guidance for the development of PROs and mandated their inclusion in clinical trials. IBD control questionnaire is one such validated questionnaire developed by Bodger et al., taking physical, social, emotional and treatment response domains into consideration. IBD control-8 subscore containing 8 items and a VAS has been developed and validated in the same study. In this study, the VAS score with cutoff of $\geq 85$ achieved $64.3 \%$ sensitivity and $90 \%$ specificity (AUC, 0.86; $P<0.001)$ and IBD control-8 identified patients in clinical re- mission with $67.5 \%$ sensitivity and $90.6 \%$ specificity (AUC, $0.90 ; P<0.001$ ), with a cutoff of $\geq 13$ points. In our study, the IBD control-8 questionnaire had similar sensitivity and specificity, but the sensitivity of the IBD control-VAS score was low (34.4\%) compared to the study by Bodger et al. ${ }^{4}$ This could be partially explained by the stringent definition of clinical remission in our study $(\mathrm{SCCAI} \leq 2)$.

There has been a paradigm shift in the therapeutic targets of IBD. ${ }^{10}$ Biomarkers of inflammation like CRP and FCP are commonly used for monitoring disease activity, and recently updated STRIDE consensus also recommends biomarkers like

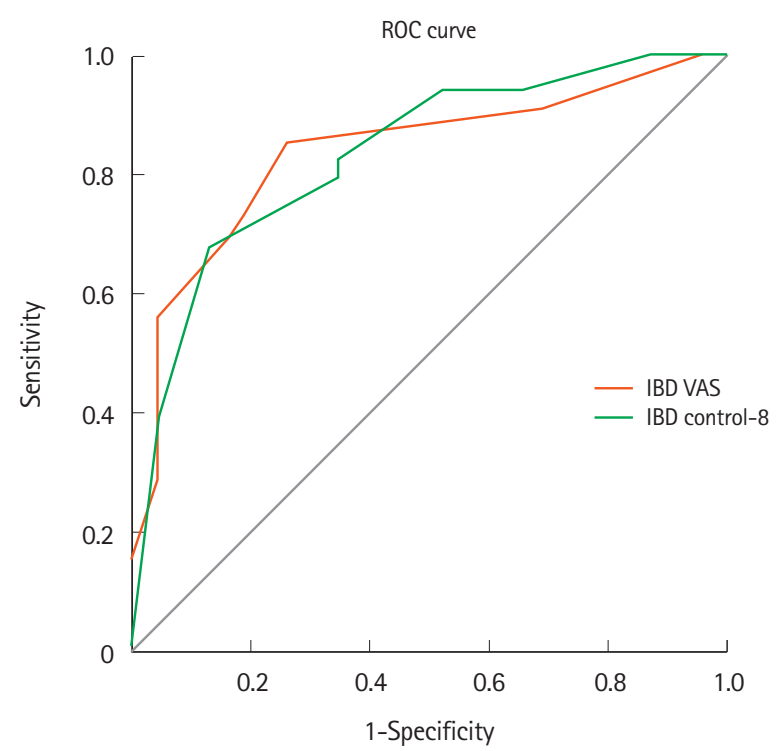

Fig. 1. Receiver operating characteristic (ROC) curve of IBD-VAS, IBD control-8 in predicting clinical remission. IBD, inflammatory bowel disease; VAS, visual analog scale. 
CRP and FCP as intermediate targets. ${ }^{10}$ FCP has been shown to be correlated with endoscopic and histological remission. However, the cutoffs vary across studies. In a metanalysis by Dulai et al.," ${ }^{11}$ the authors showed that FCP of $<50 \mu \mathrm{g} / \mathrm{g}$ has the potential to avoid $55 \%-65 \%$ endoscopies by ruling out endoscopic disease activity. A recent study also demonstrated good sensitivity and specificity of FCP with a cutoff of $157.5 \mathrm{mg} / \mathrm{kg}$ in predicting mucosal remission following biological therapy. ${ }^{12}$ A phase 2 study by Sandborn et al. ${ }^{13}$ showed an FCP cutoff of $150 \mathrm{mg} / \mathrm{kg}$ achieved a sensitivity of $79 \%$ and specificity of $75 \%$ in detecting endoscopic remission in patients treated with tofacitinib. In our study, FCP less than $150 \mu \mathrm{g} / \mathrm{g}$ has shown good diagnostic accuracy in identifying patients in clinical remission with sensitivity and specificity of $81.3 \%$ and $88 \%$, respectively.

FCP correlates well with endoscopic and histological remission, better than clinical symptoms. In a study by Lobatón et al., ${ }^{14}$ FCP better correlated with endoscopic remission than clinical remission assessed based on Mayo score ( $r=0.72$ vs. $r=0.63)$. In another study with small sample $(\mathrm{n}=39)$ there was poor correlation of FCP with SCCAI score $(r=0.2)$ but strong correlation with UCEIS $(r=0.7)$ and histological index $(r=0.9){ }^{15}$ PRO2 comprising rectal bleeding and stool frequency has been accepted as interim PRO till validated better scores are available. There is only one study published as an abstract form by Samaan et al., ${ }^{16}$ which validated PRO2 against FCP, SCCAI and IBD control score in patients of UC receiving golimumab as a part of GO-LEVEL study. In this study, authors demonstrated strong correlation between PRO2 and SCCAI $(r=0.94, P<0.001)$, as well as with IBD control $(r=-0.82, P<0.001)$ and IBD control-VAS $(r=-0.78, P<0.001)$. Significant correlations were also seen between PRO2 and FC $(r=0.38, P<0.001)$ as well as PRO2 and CRP $(r=0.31, P<0.001)$. Our study has demonstrated a moderate correlation among FCP, IBD control-VAS, and IBD control-8 subscores, and FCP correlated better with SCCAI score than IBD control subscores. However, our study has several limitations, including a small sample size, a lack of endoscopic and histopathological correlation, and a cross-sectional design.

Noninvasive biomarker FCP correlates moderately with PRO measures (IBD control-8 and IBD control-VAS).

\section{ADDITIONAL INFORMATION}

\section{Funding Source}

The authors received no financial support for the research, authorship, and/or publication of this article.

\section{Conflict of Interest}

No potential conflict of interest relevant to this article was reported.

\section{Author Contribution}

Conceptualization: Kamat N, Kedia S, Makharia G, Ahuja V. Data curation: Kamat N, Kante B, Kumar P, Ranjan MK, Singh MK, Kumar S, Sachdev V. Formal analysis: Kamat N, Vuyyuru SK, Kedia S, Sahu P, Kante B. Investigation: Singh MK, Kumar S, Sachdev V. Methodology: Kamat N, Vuyyuru SK, Kedia S, Makharia G. Supervision: S Kedia, Makharia G, Ahuja V. Validation: Vuyyuru SK. Visualization: Kumar P, M Ranjan, Singh MK, Kumar S, Sachdev V. Writing - original draft: Kamat N, Vuyyuru SK, Kedia S, Sahu P. Writing - review \& editing: Kamat N, Vuyyuru SK, Kedia S, Sahu P, Ahuja V. Approval of final manuscript: all authors.

\section{ORCID}

Kamat N

Vuyyuru SK

Kedia S

Sahu P

Kante B

Kumar P

Ranjan MK

Singh MK

Kumar S

Sachdev V

Makharia G

Ahuja V

https://orcid.org/0000-0003-3504-7290 https://orcid.org/0000-0002-8281-3237 https://orcid.org/0000-0002-5758-0144 https://orcid.org/0000-0002-9847-0136 https://orcid.org/0000-0001-5363-7273 https://orcid.org/0000-0001-5630-8715 https://orcid.org/0000-0003-2575-5971 https://orcid.org/0000-0001-6152-0139 https://orcid.org/0000-0001-5398-1330 https://orcid.org/0000-0003-0143-7653 https://orcid.org/0000-0002-2474-2194 https://orcid.org/0000-0002-1577-0118

\section{Supplementary Material}

Supplementary materials are available at the Intestinal Research website (https://www.irjournal.org).

\section{REFERENCES}

1. Graff LA, Walker JR, Lix L, et al. The relationship of inflammatory bowel disease type and activity to psychological functioning and quality of life. Clin Gastroenterol Hepatol 2006;4:14911501.

2. Burke LB, Kennedy DL, Miskala PH, Papadopoulos EJ, Trentacosti AM. The use of patient-reported outcome measures in the evaluation of medical products for regulatory approval. 
Clin Pharmacol Ther 2008;84:281-283

3. Williet N, Sandborn WJ, Peyrin-Biroulet L. Patient-reported outcomes as primary end points in clinical trials of inflammatory bowel disease. Clin Gastroenterol Hepatol 2014;12:12461256.

4. Bodger K, Ormerod C, Shackcloth D, Harrison M; IBD Control Collaborative. Development and validation of a rapid, generic measure of disease control from the patient's perspective: the IBD-control questionnaire. Gut 2014;63:1092-1102.

5. Kim DJ, Jeoun YM, Lee DW, Koo JS, Lee SW. Usefulness of fecal immunochemical test and fecal calprotectin for detection of active ulcerative colitis. Intest Res 2018;16:563-570.

6. Higgins PD, Schwartz M, Mapili J, Krokos I, Leung J, Zimmermann EM. Patient defined dichotomous end points for remission and clinical improvement in ulcerative colitis. Gut 2005; 54:782-788.

7. Dudeja P, Bahuguna P, Singh A, Bhatnagar N. Refining a socioeconomic status scale for use in community-based health research in India. J Postgrad Med 2015;61:77-83.

8. Keller R, Mazurak N, Fantasia L, et al. Quality of life in inflammatory bowel diseases: it is not all about the bowel. Intest Res 2021;19:45-52.

9. Schreiber S, Panés J, Louis E, Holley D, Buch M, Paridaens K. Perception gaps between patients with ulcerative colitis and healthcare professionals: an online survey. BMC Gastroenterol 2012;12:108.

10. Turner D, Ricciuto A, Lewis A, et al. STRIDE-II: an update on the Selecting Therapeutic Targets in Inflammatory Bowel Dis- ease (STRIDE) Initiative of the International Organization for the Study of IBD (IOIBD). Determining therapeutic goals for treat-to-target strategies in IBD. Gastroenterology 2021;160: 1570-1583.

11. Dulai PS, Battat R, Barsky M, et al. Incorporating fecal calprotectin into clinical practice for patients with moderate-to-severely active ulcerative colitis treated with biologics or smallmolecule inhibitors. Am J Gastroenterol 2020;1 15:885-894.

12. Bertani L, Blandizzi C, Mumolo MG, et al. Fecal calprotectin predicts mucosal healing in patients with ulcerative colitis treated with biological therapies: a prospective study. Clin Transl Gastroenterol 2020;11:e00174.

13. Sandborn WJ, Panés J, Zhang H, Yu D, Niezychowski W, Su C. Correlation between concentrations of fecal calprotectin and outcomes of patients with ulcerative colitis in a phase 2 trial. Gastroenterology 2016;150:96-102.

14. Lobatón T, Rodríguez-Moranta F, Lopez A, Sánchez E, Rodríguez-Alonso L, Guardiola J. A new rapid quantitative test for fecal calprotectin predicts endoscopic activity in ulcerative colitis. Inflamm Bowel Dis 2013;19:1034-1042.

15. Walsh A, Kormilitzin A, Hinds C, et al. Defining faecal calprotectin thresholds as a surrogate for endoscopic and histological disease activity in ulcerative colitis: a prospective analysis. J Crohns Colitis 2019;13:424-430.

16. Samaan M, Cunningham G, Tamilarasan G, et al. P143 Validation of the two-item UC PRO2 against SCCAI, biochemical disease activity and quality of life. Gut 2021;70(Suppl 1):A116A117. 
See "Correlation of fecal calprotectin and patient-reported outcome measures in patients with ulcerative colitis" on pages 269-273.

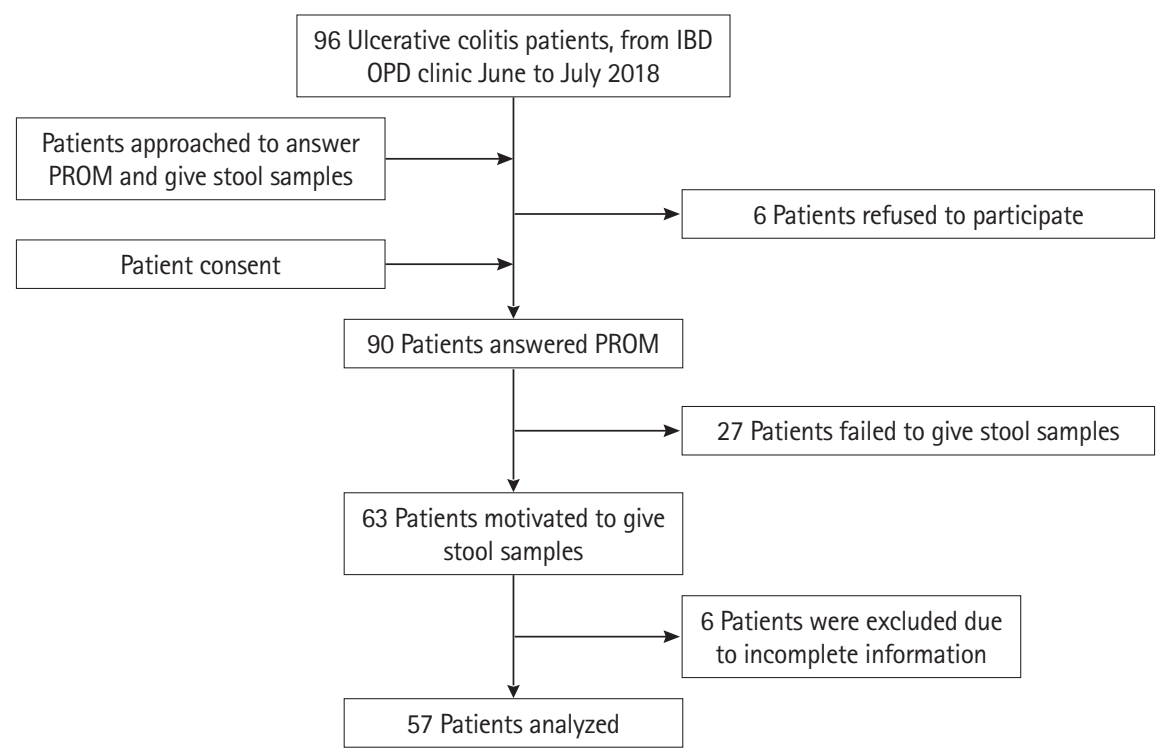

Supplementary Fig. 1. Flow diagram showing screening and recruitment. IBD, inflammatory bowel disease; OPD, outpatient department; PROM, patient reported outcome measure. 


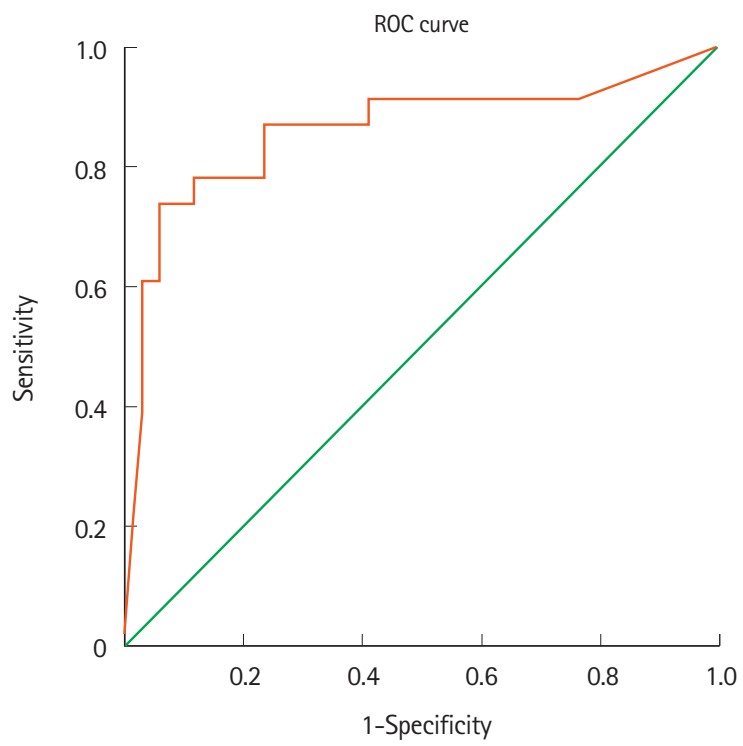

Supplementary Fig. 2. Receiver operating characteristic (ROC) curve of fecal calprotectin predicting clinical remission. 
Supplementary Table 1. IBD Control-8 Questionnaire

\begin{tabular}{l}
\hline Questionnaire \\
\hline 1. Do you believe that \\
a. Your IBD has been well controlled in the past 2 weeks \\
b. Your current treatment is useful in controlling your IBD \\
2. In the past 2 weeks did you \\
a. Miss any planned activities because of IBD \\
b. Wake up at night because of symptoms of IBD \\
c. Suffer from significant pain or discomfort \\
d. Often feel lacking in energy (fatigued) \\
e. Feel anxious or depressed because of your IBD \\
f. Think you need a change to your treatment
\end{tabular}

$\mathrm{IBD}$, inflammatory bowel disease. 
Supplementary Table 2. Correlation between FCP, SCCAI, IBD Control-VAS and Individual Components of IBD Control-8

\begin{tabular}{|c|c|c|c|c|c|c|}
\hline Variable & FCP & $P$-value & SCCAI & $P$-value & IBD-VAS & $P$-value \\
\hline Your IBD has been well controlled in the past 2 weeks? & 0.504 & 0.000 & 0.413 & 0.001 & 0.433 & 0.001 \\
\hline Your current treatment is useful in controlling your IBD? & 0.496 & 0.000 & 0.410 & 0.002 & 0.438 & 0.001 \\
\hline Miss any planned activities because of IBD? & 0.104 & 0.441 & 0.210 & 0.116 & 0.097 & 0.472 \\
\hline Wake up at night because of symptoms of IBD? & 0.376 & 0.004 & 0.520 & 0.000 & 0.298 & 0.024 \\
\hline Suffer from significant pain or discomfort? & 0.308 & 0.020 & 0.572 & 0.000 & 0.325 & 0.014 \\
\hline Often feel lacking in energy (fatigued) & 0.362 & 0.006 & 0.404 & 0.002 & 0.322 & 0.014 \\
\hline Feel anxious or depressed because of your IBD? & 0.237 & 0.077 & 0.145 & 0.282 & 0.058 & 0.668 \\
\hline Think you needed a change to your treatment? & 0.205 & 0.126 & 0.236 & 0.077 & 0.125 & 0.353 \\
\hline
\end{tabular}

FCP, fecal calprotectin; SCCAI, Simple Clinical Colitis Activity Index; IBD, inflammatory bowel disease; VAS, visual analog scale. 